\title{
SPECIFIC NATURAL BIOACTIVE TYPE 1 COLLAGEN PEPTIDES ORAL INTAKE REVERSE SKIN AGING SIGNS IN MATURE WOMEN
}

\author{
L. Duteil ${ }^{1}$, C. Queille-Roussel ${ }^{1}$, Y. Maubert ${ }^{1}$, J. Esdaile ${ }^{1}$, C. Bruno-Bonnet ${ }^{2}$, J.-P. Lacour ${ }^{1,3}$
}

\begin{abstract}
Objective: To assess the anti-aging potential of three type I fish collagen hydrolysates $(\mathrm{CH} 1=\mathrm{Naticol} 囚 \mathrm{BPMG}$, $\mathrm{CH} 2=$ Naticol ${ }^{\circledR}$ HPMG, $\mathrm{CH} 3=$ Naticol ${ }^{\circledR} 1000 \mathrm{MG}$ ) on skin aging signs for three different body sites of mature women. Design: Double-blind, randomized and Placebo-controlled clinical study. Setting: Centre of Clinical Pharmacology Applied to Dermatology (CPCAD, Nice). Participants: Sixty women aged 46-69 years having skin aging signs on the face. Intervention: Participants were randomized to receive a once daily $5 \mathrm{~g}$ dose of one of the $\mathrm{CHs}$ or Placebo for 8 weeks. Measurements: Skin biomechanics, skin hydration and visual assessment of the crow's-feet wrinkles were evaluated after 4 and 8 weeks of treatment. Subject satisfaction questionnaire and Investigator global efficacy appreciation (IGEA) were also used. Results: Skin biomechanics indicated a significant improvement of skin firmness for the three $\mathrm{CH}$ compared to Placebo, in particular for $\mathrm{CH} 2$. An increase of overall skin elasticity for $\mathrm{CH} 3(\mathrm{p}=0.017)$ and $\mathrm{CH} 2(\mathrm{p}=0.044)$ on the abdomen was also observed. This was corroborated by the significant decrease of the crow's-feet wrinkle score at week 8 for both $\mathrm{CH} 3$ and $\mathrm{CH} 2$ ( $\mathrm{p}=0.023$ and $\mathrm{p}=0.014$, respectively). Concerning the self-questionnaire, overall the number of positive responses was significantly higher for $\mathrm{CH} 2$ compared to Placebo and other $\mathrm{CHs}$. For the IGEA, the number of favorable answers was greater for $\mathrm{CH} 2$ than for the Placebo group (80\% vs. 36\%, $\mathrm{p}=0.025)$. A positive influence of $\mathrm{CH}$ treatments could be observed for skin hydration but failed to reach statistical significance. Conclusion: The tested type I fish collagen hydrolysates have beneficial effects on skin quality. In particular, $\mathrm{CH} 2$ demonstrated the greatest range of these effects including improvement of skin biomechanics, decrease of wrinkles, good subject satisfaction and no related adverse events.
\end{abstract}

Key words: Fish collagen peptides, skin elasticity, anti-aging.

\section{Introduction}

Skin aging (1) is one of the most important current dermatological concerns. There are two types of skin aging, one is intrinsic aging which is due to the passage of time (chronological) and the other is premature or extrinsic aging due to environmental aggressions. Aging may be considered as the accumulation of diverse deleterious changes in cells and tissues.

According to the free-radical theory of aging, reactive oxygen species (ROS), primarily arising from oxidative cell metabolism, play a major role in both chronological aging and photoaging (2). Cellular aging (HayflickLimit) and shortening of telomeres, mitochondrial DNA mutations, genetic mutations and decrease of certain

1. Centre of Clinical Pharmacology Applied to Dermatology (CPCAD), L'Archet 2 Hospital, Nice, France; 2. Weishardt International, Rond-point Georges Jolimaitre BP259, 81305 Graulhet, France; 3. Service de Dermatologie, L'Archet 2 Hospital, Nice, France

Corresponding Author: Luc Duteil, CPCAD (Centre of Clinical Pharmacology Applied to Dermatology), Hôpital l'Archet 2, 151 route de Saint Antoine de Ginestière, 06202 Nice, France, Phone : 00.33.4.92.03.62.45, Fax : 00.33.4.92.03.62.39, e-mail : luc.duteil@skinpharma.fr

Received September 14, 2015

Accepted for publication November 17, 2015 hormone levels are also implicated in intrinsic skin aging (3).

Extrinsic skin aging, also known as premature skin aging or photoaging, primarily arises from UV-light exposure (4). Approximately $80 \%$ of facial skin aging is attributed to UV-exposure (5). Further relevant exogenous factors are exposure to tobacco smoke, airborne particulate matter, infrared radiation, ozone and malnutrition.

Clinical signs of dermal atrophy and skin aging (including skin dryness, hypo/hyperpigmentation, diminished skin elasticity and firmness) are associated with a reduction and disorganization of collagen $(6,7)$. Scanning electron microscopic examination has shown that the number and width of collagen fibre bundles decreases with age (8).

Collagen, particularly Types 1 and 3 collagen, is the main building block of human skin $(9,10)$. It is the major structural protein present in skin and bone of all animals where it accounts for about $30 \%$ of the total protein content (11). Types 1 and 3 collagen are synthesized from precursor molecules called procollagens which are derived from dermal fibroblasts. Commercially, 
collagen is mainly sought-after for the production of gelatin, a high value functional protein due to its unique gel-forming capacity. Gelatin is the most popular hydrocolloid in current use (12). Despite having a low biological value, gelatin, like collagen, is widely used in pharmaceutical and medical applications because of its biodegradability and biocompatibility in the physiological environment as well as being used in many technical applications (13).

However, some factors recognized as a serious risk for human health, such as the outbreak of bovine spongiform encephalopathy (BSE) and hoof-and-mouth disease (FMD) (Aphtae epizooticae) which are both transmissible via food, have severely hampered the use of by-products of bovine and porcine origin (14).

Due to similar rheological behaviours, gelatin from warm water fish can be a good alternative to that derived from pigs (15), which is considered to be the best among all gelatins due to its higher proline and hydroxyproline content (11).

In the recent years, the relationship between nutrition factors and skin function has received increasing attention. Animal experiments and preclinical human trials investigating the effects of oral supplementation with collagen peptides have indicated the possibility that dietary compounds can modulate skin function (16-21). For example, Matsuda et al. (18) reported that in pigs a 9-week oral ingestion of collagen hydrolysates induced increased fibroblast density and enhanced formation of collagen fibrils in the dermis in a protein-specific manner. It has also been reported that a daily ingestion of collagen hydrolysates $(10 \mathrm{~g})$ and vitamin $C(0,4 \mathrm{~g})$ improved the skin hydration of 20 healthy Japanese women compared to a Placebo group. The results of this study suggested that vitamin $C$ enhanced the effect of the collagen (16).

In a pilot trial (not double-blind and not Placebocontrolled study), an oral daily ingestion of $5 \mathrm{~g}$ of hydrolysed fish collagen mixed with vitamin C $(50 \mathrm{mg})$ and glucosamine $(60 \mathrm{mg})$ improved skin hydration and elasticity on face and forearms of female (19). In a double-blind and Placebo-controlled study, Proksch et al. (22) reported on the effects of specific porcine collagen peptides $(2,5 \mathrm{~g} /$ day and $5 \mathrm{~g} /$ day) on face skin assessed as dry.

Up until now, there have been very few randomized, double-blind, placebo-controlled studies of daily-dose type 1 fish collagen peptides in the treatment of aging signs in women. Additionally, the study here reported evaluates the effect of collagen peptides on several different skin locations rather than one single site.

In contrast to those studies which have been published where collagen is assessed in conjunction with other bioactive compounds (e.g. vitamin C, hyaluronic acid ...), or is derived from a mixture of sources (e.g. porcine, bovine or piscine) the present study has used hydrolyzed collagen of exclusively piscine origin without additional active ingredients.
Hydrolyzed collagen is one of the foremost health ingredients in Asia. It is the most popular and the most used in the Japanese food processing industry for the manufacture of FOSHU (Food for Specified Health Use) products. The "health" claims for hydrolyzed collagen are mainly related to skin and hair beauty and bone and joint health. In Asian countries generally, collagen has been used traditionally and is also recognized for its benefits on skin, hair, and bone and joint health. In the USA, according to the regulation of the FDA (Food and Drug Administration) on food ingredients, collagen hydrolysates are considered as GRAS (Generally recognized as safe).

This present double-blind, Placebo-controlled and randomised study was intended to assess the antiaging potential of three specific collagen hydrolysates with different peptide profiles and molecular weights on the aging signs of the skin of mature women. The hydrolysates or peptides which were the object of this cosmetic efficacy study are commercialized products (Naticol®, Weishardt) derived from fish skins suitable for use in human food.

\section{Materials and methods}

\section{Study Design}

This study was conducted in accordance with the ethical principles originating from the Declaration of Helsinki and its amendments, the ICH guidelines for Good Clinical Practice (GCP) as currently in force and in compliance with local regulatory requirements. The protocol was approved by the Ethic Committee of Nice (France). Informed consent was obtained from all subjects before the initiation of the study. The study was carried out as a monocentric, double-blinded, randomized, placebo-controlled supplementation study on the effects of three collagen hydrolysates (Naticol®) on skin elasticity (primary criterion), skin wrinkles and skin hydration after 8 weeks of daily intake.

\section{Subjects}

Sixty (60) healthy female subjects with skin type II to $\mathrm{V}$ according to the Fitzpatrick scale, meeting specific criteria of inclusion/non-inclusion were to be included in the study and split into four homogeneous groups of 15 women each. They were to be randomly assigned at the CPCAD (Centre de Pharmacologie Clinique Appliquée à la Dermatologie), Nice University Hospital, France, to receive either a $\mathrm{CH}$ (specific fish collagen peptides, Naticol ${ }^{\circledR}$, Weishardt France) once daily or matching Placebo, maltodextrin for 8 weeks. Trial products were prepared by the manufacturer.

The main inclusion criteria were the following: Healthy female subjects, 45 to 70 years old with skin type II to V, 
having skin aging signs on the face with periorbital lines of medium to moderate severity corresponding at least to level 3 of the classification of Lemperle (23), agreeing to take an oral supplementation during 8 consecutive weeks and willing to comply with the study restrictions and complete the study phase. Main exclusion criteria were: subjects who had been treated with cosmetic or medical anti-aging treatments during the 8 weeks preceding the inclusion or surgical antiaging treatments during the preceding 8 months; subjects having a known allergy to fish; subjects who had been sunburned in the last month; subjects taking any dietary supplement which could interfere with the study results (e.g. collagen supplementation) or which could modify the bioavailability of the tested product (e.g.: hypo-caloric diet, or high protein diet, etc...).

\section{Test products}

Tested products (fish collagen peptides, Naticol@, Weishardt International Group) and molecular weights ( $\mathrm{mw}$, specific peptide profile) were the following: Naticol ${ }^{\circledR}$ BPMG $(\mathrm{CH} 1, \mathrm{mw}=2 \mathrm{kDa})$, Naticol ${ }^{\circledR}$ HPMG $(\mathrm{CH} 2, \mathrm{mw}=4 \mathrm{kDa})$, Naticol ${ }^{\circledR}$ 1000MG $(\mathrm{CH} \mathrm{3,} \mathrm{mw}=$ $2 \mathrm{kDa})$

Dose: one daily intake of a $5 \mathrm{~g}$ sachet in $20 \mathrm{cl}$ of liquid 15 minutes before breakfast, for 8 weeks.

The Placebo (maltodextrin) was manufactured by Tereos-Syral (France) and provided to the Investigator in boxes containing $5 \mathrm{~g}$ single dose sachets.

\section{Assessment times}

There were 3 assessment times: immediately before starting the product intake (baseline or week0), after 4 weeks (week4) and after 8 weeks (week8) of daily product intake. The volunteer's compliance and product tolerance were checked after 4 and 8 weeks of intake.

\section{Skin Biometry measurements}

Skin biometry measurements were performed on three body sites: on the face (bony part of the cheek), on the volar aspect of the dominant forearm and on the abdomen $(10 \mathrm{~cm}$ to one side of the navel).

\section{Skin biomechanics}

The cutometer SEM 575 Skin Elasticity Meter (Courage and Kazhaka, Köln, Germany) allows measurement of the deformation perpendicular to the skin surface using a suction method $(24,25)$. A negative pressure of 400 mbars is applied to the skin through the probe for a period of 2 seconds, followed by a relaxation period of 2 seconds. This cycle is repeated 5 times. The deformation induced on the skin is measured by an optical system placed in the probe. The probe aperture was $2 \mathrm{~mm}$ diameter for the face and forearm measurements and $6 \mathrm{~mm}$ for the abdomen. The measured parameters are (see Fig. 1): Uf the total distensibility of the skin; Ue, the elastic deformation; Ur, the elastic back deformation; $U a$, the total deformation recovery; Ua/Uf and $\mathrm{Ur} / \mathrm{Ue}$ the gross and the pure elasticity respectively. Three measurements of 5 cycles were performed on each test zone and the results averaged for analysis.

\section{Skin hydration}

Skin hydration measurements were performed using the corneometer CM 825 (Courage \& Khazaka, Köln, Germany). This instrument allows measurement of the hydration state of the first horny layers using electrical capacitance measurements $(26,27)$. Once the probe is placed perpendicularly on the skin surface, measurements are taken in less than 1 second. Three measurements were taken on each site, and the results averaged for analysis.

\section{Clinical assessments}

Score of peri-orbital wrinkles (crow's feet) was assessed clinically by the investigator at the week 4 and week 8 visits using a 10-point photographic scale with: 0 $=$ no wrinkles, $9=$ numerous deep coarse wrinkles (28).

\section{Investigator Global Efficacy Appreciation}

The global efficacy (IGEA) was assessed by the Investigator at week8. The question "Would you say that the treatment which has been tested is globally efficient for the treatment of aging signs" was answered using the following 5 point scale: Agree totally, Agree partially, No opinion, Disagree partially, Disagree totally.

\section{Self-questionnaire}

At week8, a questionnaire was used to evaluate how satisfied the volunteers were with the treatment in terms of the decrease of skin wrinkles, as well as the increase of homogeneity of tone, skin hydration, firmness and brightness.

At each visit, systemic side effects such as gastrointestinal discomfort, diarrhea, headache, skin rash or other symptoms were recorded.

\section{Statistical analysis}

The statistical analyses were performed using the $\mathrm{R}$-Software version 2.15.2. Prior to any statistical analysis, the normality of each variable was tested using the Shapiro Wilks test of normality. For each group, a timeanalysis of raw data was performed using a mixed- 
Table 1

Demographic data by treatment group

\begin{tabular}{|c|c|c|c|c|c|c|c|}
\hline & & $\begin{array}{c}\text { All } \\
\mathrm{N}=60\end{array}$ & $\begin{array}{c}\text { Naticol }^{\circledR} \\
\text { 1000MG } \\
\mathrm{N}=15\end{array}$ & $\begin{array}{c}\text { Naticol }^{\circledR} \\
\text { BPMG } \\
\text { N=15 }\end{array}$ & $\begin{array}{c}\text { Naticol }{ }^{\circledR} \\
\text { HPMG } \\
\text { N=15 }\end{array}$ & $\begin{array}{c}\begin{array}{c}\text { Placebo } \\
\text { N=15 }\end{array}\end{array}$ & $\begin{array}{l}\text { Comparative } \\
\text { analysis* }\end{array}$ \\
\hline \multirow[t]{3}{*}{ Age (years) } & Mean & $57 \pm 6$ & $56 \pm 5$ & $55 \pm 8$ & $59 \pm 7$ & $56 \pm 7$ & $\mathrm{p}=0.316(\mathrm{~ns})$ \\
\hline & Min & 46 & 46 & 55 & 48 & 46 & \\
\hline & Max & 69 & 66 & 66 & 69 & 67 & \\
\hline \multirow[t]{3}{*}{ Weight (kg) } & Mean & 67 & $69 \pm 14$ & $68 \pm 9$ & $68 \pm 12$ & $64 \pm 13$ & $\mathrm{p}=0.606(\mathrm{~ns})$ \\
\hline & Min & 48 & 48 & 54 & 48 & 49 & \\
\hline & Max & 98 & 98 & 86 & 85 & 95 & \\
\hline \multirow[t]{3}{*}{ Height $(\mathrm{cm})$} & Mean & $163 \pm 5$ & $164 \pm 5$ & $161 \pm 6$ & $164 \pm 4$ & $163 \pm 6$ & $\mathrm{p}=0.378(\mathrm{~ns})$ \\
\hline & Min & 151 & 153 & 152 & 155 & 151 & \\
\hline & Max & 173 & 170 & 170 & 170 & 173 & \\
\hline \multirow[t]{2}{*}{ Skin type } & II & $5(8.3 \%)$ & $1(1.7 \%)$ & $2(3.3 \%)$ & $1(1.7 \%)$ & $1(1.7 \%)$ & \\
\hline & III & $55(91.7 \%)$ & $14(23.3 \%)$ & $13(21.7 \%)$ & $14(23.3 \%)$ & $14(23.3 \%)$ & \\
\hline
\end{tabular}

design ANOVA with the variable "Subject" as random factor and the variable "Visit" as fixed factor. Multiple post-hoc comparisons were done using the Dunnett method to compare each time-point (week4 and week8) with baseline. If the residuals of the ANOVA were not normally distributed then a Friedman test was carried out and pairwise comparisons were performed using a Wilcoxon signed rank test with a Bonferroni adjustment.

At each time-point (week4 and week8), the 4 groups of treatment were compared on the differences week4week 0 and week8-week 0 using an analysis of variance with one factor "Group" followed by a Tukey's multiple comparison test. If the normality of the distribution (w0wi) of one or both groups was rejected, the comparison of the 2 groups were performed using a Kruskal-Wallis test and pairwise comparisons for factor "Group" were performed using a Mann-Whitney adjusted test.

For the questionnaire, positive answers (i.e. "Agree totally" and "Agree partially") on one side and negative answers (i.e. "Disagree totally" and "Disagree partially") on the other side were merged. At each time-point, the 4 groups were compared using a $\mathrm{Chi}^{2}$ test or a Fischer exact test if the number of subjects in one category was lower or equal to 5 .

\section{Results}

All volunteers but one completed the 8-week study. One serious adverse event (SAE) was reported in the Placebo group. This SAE (femoral neck fracture) led to the discontinuation of the study after visit week 4 and was not related to the tested product. The four groups were homogenously distributed according to age and skin type (Table I). The mean age was $57 \pm 7$ years old (range 46 to
69 years) and most of the subjects were skin type III (92\%) and menopaused $(72 \%)$.

\section{Skin biomechanics}

There was no significant difference in skin biomechanics parameters between the treatment groups and the Placebo prior to the product intake $(\mathrm{p}>0.05)$ whatever the body site.

Significant results concerning biomechanics parameters were observed at week 4 and week 8 for the Naticol® groups and not for the Placebo group. Globally, significant variations compared to baseline were the following, after 8 weeks:

- a decrease of the skin distensibility (Uf) on the forearm only by $10 \%$ for $\mathrm{CH} 3(\mathrm{p}=0.002)$ and $9,2 \%$ for $\mathrm{CH} 1(\mathrm{p}=$ 0.044). This indicates firmer forearm skin (Fig. 2).

- an increase of the ability to return to the original state (Uf - Ua) (increase of skin firmness) for $\mathrm{CH} 1$ ( $\mathrm{p}=$ $0.015)$ on the forearm and $\mathrm{CH} 2(\mathrm{p}=0.037)$ on the face.

- an increase of the overall elasticity (Ua/Uf) (increase of gross elasticity) for $\mathrm{CH} 3(\mathrm{p}=0.021)$ and $\mathrm{CH} 2(\mathrm{p}=$ 0.024) on the abdomen.

- a decrease of the last maximum amplitude (R3) (increase of skin firmness) on the forearm only for both $\mathrm{CH} 3(\mathrm{p}=0.014)$ and $\mathrm{CH} 1(\mathrm{p}=0.027)$.

- a decrease of the last minimum amplitude (R4) (increase of skin firmness) on the abdomen only, by $21 \%$ for $\mathrm{CH} 3(\mathrm{p}=0.001)$, by $21,8 \%$ for $\mathrm{CH} 2(\mathrm{p}<0.001)$ and by $13,85 \%$ for $\mathrm{CH} 1(\mathrm{p}=0.039)$.

- a decrease of the residual deformation at the end of the measuring cycle (R9) (increase of skin firmness) on the face by $20,4 \%$ for $\mathrm{CH} 3(\mathrm{p}=0.004)$ and $\mathrm{CH} 2(\mathrm{p}=0.002)$ and by $18,2 \%$ for CH1 (p=0.001) (Fig. 3). 
- An increase of the viscoelastic ratio $(\mathrm{Uv} / \mathrm{Ue})$ on the face $(p=0.035)$ can also be noted for the Placebo (negative effect) only and no significant effect for the CHs.

\section{Figure 1}

Skin deformation curve of the cutometer as a function of time

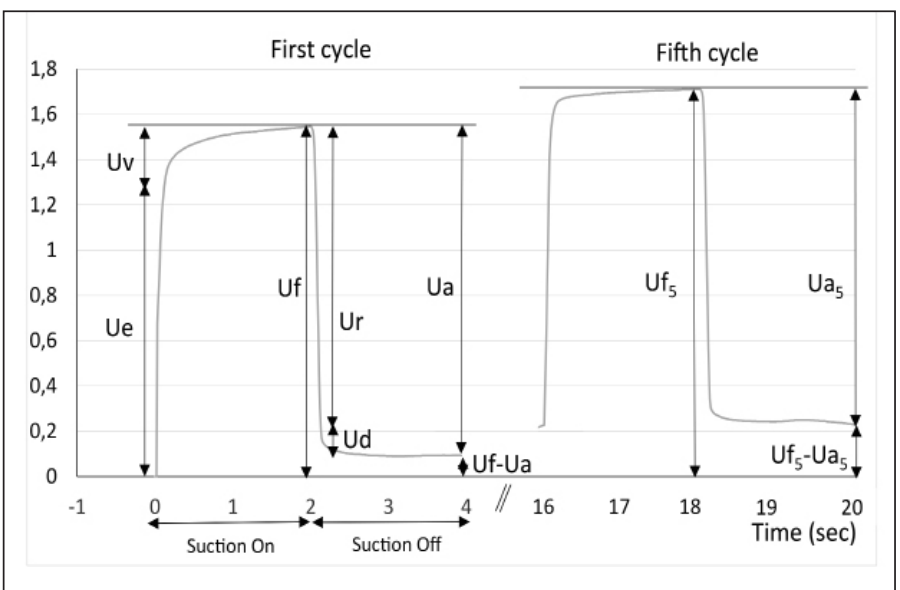

Figure 2

Evolution of skin distensibility (Uf) on forearm as a function of treatments. Both Naticol®1000MG and Naticol®BPMG induced a significant decrease of Uf, indicating an increase of skin firmness (mean \pm SEM, *: $\mathrm{p}<0.05)$.

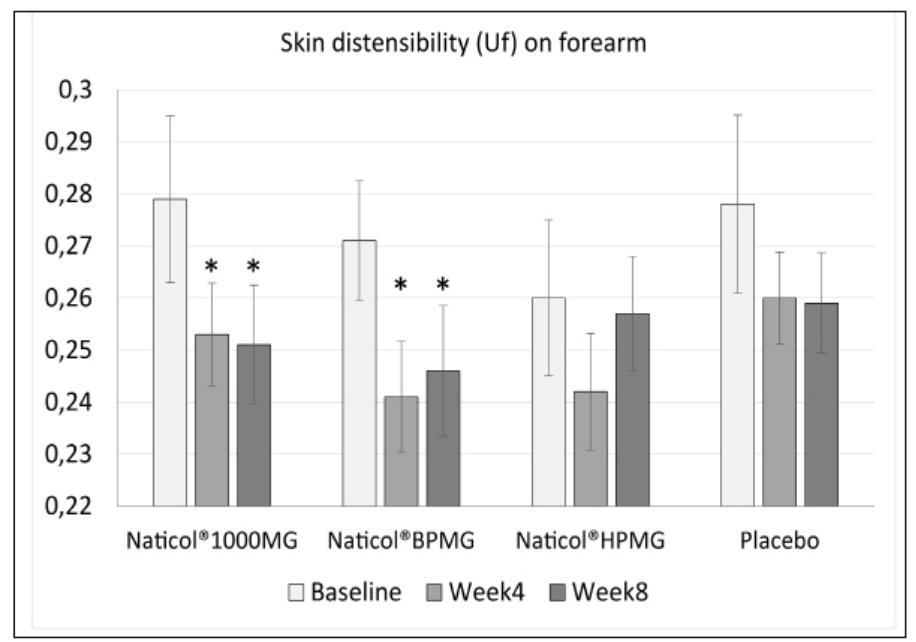

\section{Skin hydration}

There was no significant difference in skin hydration between the treatment groups and the Placebo prior to the products intake ( $p>0.05)$ whatever the body site. After 4 and 8 weeks of daily intake, no significant differences were observed between the $\mathrm{CH}$ s and the Placebo. No significant variations from baseline were observed for the $\mathrm{CHs}$ whereas a significant decrease
$(-14 \%)$ of the hydration level on the forearm was detected at week $4(\mathrm{p}<0.017)$ and week8 $(\mathrm{p}<0.018)$ for the Placebo group. Since the study was performed in winter with a decrease of temperature between baseline and week8, this could indicate a protective effect of $\mathrm{CHs}$ products on skin dehydration caused by cold weather conditions.

Figure 3

Evolution of the residual deformation (Uf5-Ua5) on the face as a function of treatments (mean \pm SEM, * $\mathrm{p}<0.01$ )

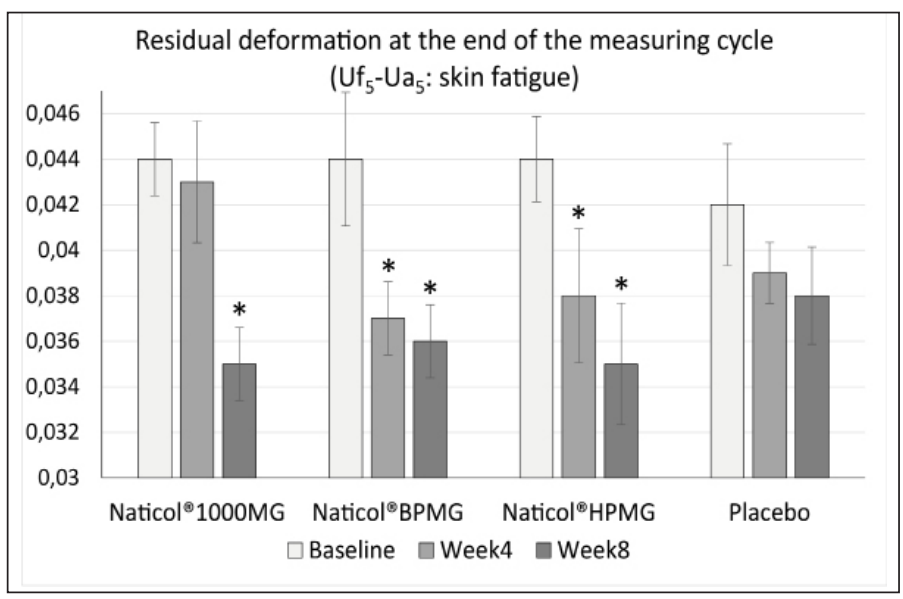

Figure 4

Variations from baseline for the mean of the clinical score of peri-orbital wrinkles $\left({ }^{*}: \mathrm{p}<0.05\right)$

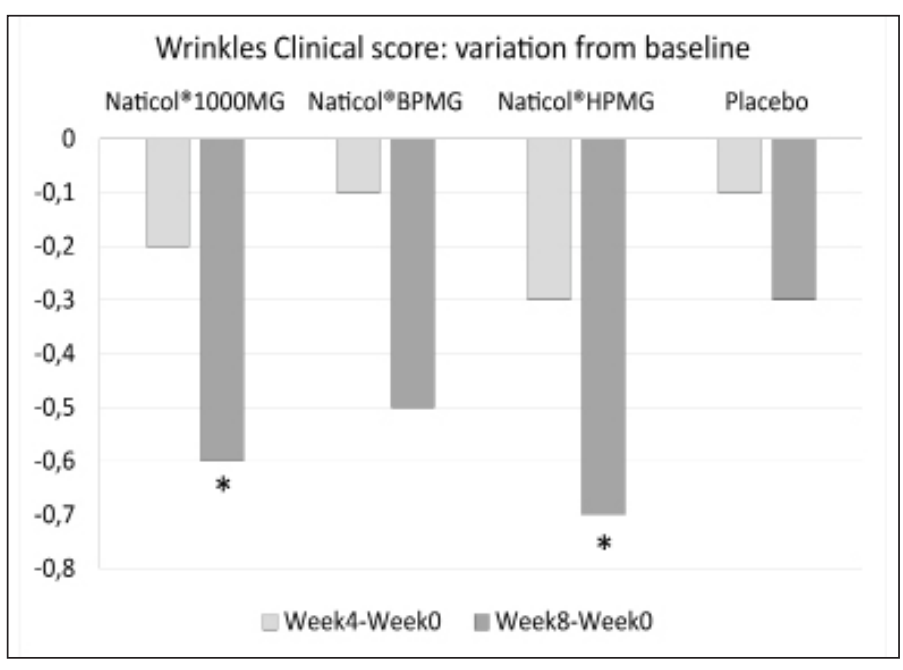

\section{Clinical assessments}

Concerning the clinical assessment of the peri-orbital wrinkles, compared to baseline, the wrinkle score was significantly decreased of $-11 \%$ at week 8 for both $\mathrm{CH} 2$ and $\mathrm{CH} 3$ ( $\mathrm{p}=0.014$ and $\mathrm{p}=0.023$, respectively, see Fig. 4). There was no significant difference between treatment groups. 


\section{Investigator global efficacy Appreciation}

Concerning the global efficacy assessed by the Investigator (IGEA), no significant difference was observed between CHs. However, the number of favorable answers for $\mathrm{CH} 2$ was significantly greater than for the Placebo group ( $80 \%$ vs. $36 \%, p=0.025$, see Fig. 5).

\section{Figure 5}

Investigator Global Assessment, distribution of response as a function of treatments at week8. The number of favorable answers for $\mathrm{CH} 2$ was significantly higher than for Placebo ( $80 \%$ vs. 36\%, p= 0.025)

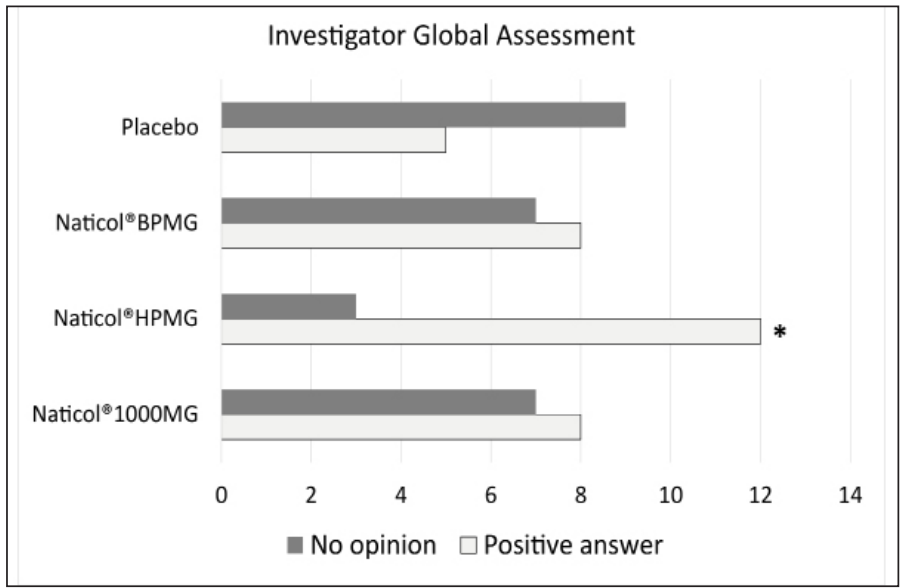

Figure 6

Distribution of responses to the self-questionnaire at week8. The number of positive responses was significantly higher for Naticol®HPMG compared to placebo and other $\mathrm{CHs}\left({ }^{* *}: \mathrm{p}<0.001\right)$

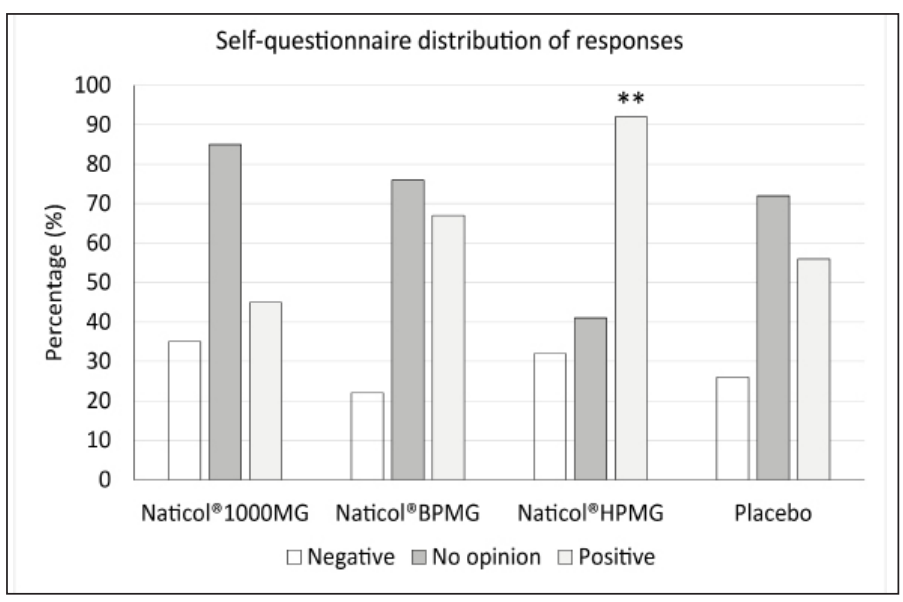

\section{Self-questionnaire}

The distribution of answers to the subject selfquestionnaire indicate that, overall, the percentage of positive answers was greater in the group $\mathrm{CH} 2$ for most of the questions (Fig. 6). Globally, when all answers were combined, the number of positive responses was significantly higher for $\mathrm{CH} 2$ compared to placebo and other CHs.

Treatment compliance was satisfactory for all groups. Overall, the mean exposure to the tested products was 56.8 days (range 51 to 58). The mean extent of exposure was approximately the same for the 4 groups.

The tolerance of the tested products was globally excellent.

\section{Discussion}

This pilot study was intended to assess the anti-aging potential of three collagen hydrolysates at three different molecular weights on the aging signs of the skin of mature women volunteers. The subjects had to take a $5 \mathrm{~g}$ sachet daily of either one $\mathrm{CH}$ or Placebo (maltodextrine) in $20 \mathrm{cl}$ of liquid 15 minutes before the breakfast, for 8 weeks.

Cutaneous aging combines intrinsic aging and photoaging. Intrinsic aging is characterized by skin roughness, wrinkles, laxity, decrease of skin thickness, loss of skin elasticity, pigmentary changes and benign tumours (1). Photoaging is the result of chronic exposure to ultraviolet (UV) light which induces elastosis (yellow discoloration and pebbly surface), telangiectasia and pre-cancerous lesions such as actinic keratosis and also malignant tumours (2). The most consistent histological change (29) in intrinsic aging is epidermal atrophy, flattening of the dermo-epidermal junction with effacement of rete pegs and deposition of pseudoxanthoma-elasticum-like papillary dermal elastolysis. In contrast, acanthosis in addition to loss of polarity, cellular atypia and deposition of tropoelastin and fibrillin are frequently discovered in photoaging skin. Glycosaminoglycans and proteoglycans are also increasingly found in photodamaged skin but fibrillin and collagen are not increased in the upper dermis (30, 31).

Photoaging is the superimposition of photodamage on intrinsically aged skin generally bringing about premature aging. This specific damage occurs by chronic (multiple) exposure of the skin to UV light. Clinically, the skin becomes coarse; epidermis thickens (hyperplasia) initially and then thins (atrophy), there is laxity, sallowness with wrinkles, irregular hyperpigmentation, lentigines, and telangiectasias $(32,33)$.

It can be assumed that the most prominent physiological alterations in chronological and photoaging are localized in the dermis and caused by the metabolism of dermal collagen fibres.

Minaguchi et al. (34) investigated in an in vitro study the effects of two doses $(0.2 \mathrm{~g} / \mathrm{kg}$ and $1.0 \mathrm{~g} / \mathrm{kg}$ body weight) of $\mathrm{CH}$ daily ingestion on the extracellular matrix of rabbit Achilles tendon, for 56 days. Both the size of collagen fibrils and the amount of glycosaminoglycans were measured in comparison with those in a rabbit fed with a control protein, lactoalbumin, or water alone. 
Ingestion of $\mathrm{CH}$ or lactoalbumin induced a significant increase in collagen fibril diameter and decrease in fibril density except for a high dose of lactoalbumin compared with the water control.

The protective effects of two gelatine hydrolysates extracted from Pacific cod skin against ultraviolet radiation-induced skin photoaging in mice were investigated (35) by measurement of the activities of superoxide dismutase (SOD), glutathione peroxidase (GSH-Px), catalase (CAT) and the glutathione (GSH), malondialdehyde (MDA) and hydroxyproline (HYP) content of photoaging skin tissue. In the hairless mouse model, results indicated that UV radiation induced a decrease in the antioxidase activity and a depletion of reduced glutathione $(20.4 \%)$ in the skin. Compared with the untreated mice, both gelatine hydrolysates can enhance the activities of SOD, GSH-Px, CAT, increase GSH and HYP content and reduce the content of MDA, which reduces skin photodamage. Moreover, the results of a histology study confirmed that both polypeptides could protect collagen fibres in skin.

During the last decade, several in vitro and in vivo animal studies have shown that collagen peptides (CP) may improve skin appearance and functions. In these studies various dermal features have been assessed such as fibroblast growth $(18,34,36,37)$, dermal extracellular matrix synthesis $(18,34)$, antioxidative protection $(38,39)$, increased angiogenesis and wound healing $(40,41)$ and anti-aging properties $(42,43)$.

To date, very few double-blind, randomized and Placebo controlled studies have been performed. Most of the clinical studies investigating $\mathrm{CH}$ effect on aging skin have been open labelled studies. Moreover, most of the studies performed involved the assessment of combined formulations containing $\mathrm{CH}$ and a mixture of several other ingredients such as hyaluronic acid, vitamins and or minerals.

The clinical effects on skin properties of daily ingestion $(5 \mathrm{~g})$ of fish type 1 collagen hydrolysate mixture were investigated during 6 weeks by Matsumoto et al. (19) on 25 Japanese women with dry skin. The results of this open-label study indicated that compared to baseline, skin hydration was significantly increased on the forearms and the back of neck but not on the face. Nevertheless, skin viscoelastic properties were significantly improved and subject's skin symptoms were 60\%-100\% improved.

In a Placebo controlled study, Sumida et al. (16) evaluated the effect of daily ingestion of $\mathrm{CH}(10 \mathrm{~g})$ on the skin hydration of 20 healthy Japanese women compared to a Placebo group (19 volunteers). A gradual improvement of water absorption capacity was observed over 60 days in volunteers who ingested $\mathrm{CH}$ in comparison with the Placebo group. However, the improvement was not statistically significant between groups. It is important to emphasize that the supplement that was administered to both the groups in this study also contained $0.4 \mathrm{~g}$ of vitamin $\mathrm{C}$. The results of this study suggested that vitamin $C$ enhanced the effect of the collagen.

In a recent double-blind Placebo controlled study (22), Proksch et al assessed the effectiveness of collagen hydrolysate $(\mathrm{CH})$, derived from a special hydrolysis of porcine type I collagen, on skin biophysical parameters related to cutaneous aging. In this trial, 69 women aged 35-55 years were randomized to receive $2.5 \mathrm{~g}$ or $5.0 \mathrm{~g}$ of $\mathrm{CH}$ or Placebo once daily for 8 weeks, with 23 subjects being allocated to each treatment group. The results clearly revealed that both dosages of the specific $\mathrm{CH}$ had a beneficial effect on skin physiology, as indicated by increased skin elasticity after 4 weeks of daily consumption. The observed effect was statistically significant ( $\mathrm{p}<0.05$ ) after 4 and 8 weeks in the treatment groups compared to Placebo. Skin roughness measured by image analysis of skin imprints and measurements of trans-epidermal water loss did not detect any significant improvements. Moreover, as for our study, no significant change in skin hydration was observed for both $\mathrm{CH}$ dosages. An interesting point revealed in our study is that a significant decrease of hydration level $(-14 \%)$ on the forearm was observed at week 4 and week 8 for the Placebo group only. Since the study was performed in winter with a decrease of temperature between baseline and week8, this could indicate a protective effect of Naticol ${ }^{\circledR}$ products on skin dehydration caused by cold weather conditions. To the best of our knowledge, a significant effect of $\mathrm{CH}$ treatment on skin hydration level measured by a noninvasive technique has never been clearly detected.

Skin elasticity is a very important marker for skin aging assessment and its associated treatments. In the present study significant effects have been observed concerning skin biomechanics. An increase of the gross elasticity (Ua/Uf) was detected on the abdomen skin for $\mathrm{CH} 3$ and $\mathrm{CH} 2$. Gross elasticity $\mathrm{Ua} / \mathrm{Uf}$ has been found to decrease with age (25) and has been proven to be the most suitable parameter (with $\mathrm{Ur} / \mathrm{Uf}$ the ratio of elastic recovery to distensibility) in detecting age-related skin alterations. Therefore, increasing this parameter could be considered as a sign of treatment efficacy. The last minimal lowest point of the last curve (R4) was shown to be positively correlated with age (24). Our results indicated a significant decrease of R4 (increase of skin firmness) on the abdomen skin for the three $\mathrm{CHs}$.

The clinical assessment of the peri-orbital wrinkles at week 8 , indicated a significant decrease of $-11 \%$ compared to baseline, for both $\mathrm{CH} 2$ and $\mathrm{CH} 3$. In a pilot openlabel human study examining the effect hydrolyzed collagen type II and low-molecular-weight hyaluronic acid and chondroitin sulfate ingestion on skin aging, the authors (44) found at week12 a significant improvement of facial lines and wrinkles with an average reduction of $13.2 \%$ from baseline but failed to detect significant effect on the the peri-orbital wrinkles. In the study by 
Proksch et al (22), the skin roughness was assessed by analysis of silicone imprints of the forearm skin using a fringe projection system (PRIMOS Compact). Over the treatment period of 8 weeks, the $\mathrm{CH}$ treated group showed no visible changes in skin roughness whereas for the Placebo group these changes were detectable.

Another open-label study (32) investigating the effects of a collagen type I hydrolysate mixture, demonstrated a weak but significant improvement of some skin surface parameters, measured using the Visioscan system (image analysis system), after 6 weeks of supplementation.

The results of these and other studies indicate that daily supplementation of collagen hydrolysates probably needs to be extended to a period of 4 to 6 months in order to detect more pronounced effects on wrinkles and skin relief.

This pilot 2-month treatment once daily study demonstrates that the collagen hydrolysate based products (Naticol ${ }^{\circledR}$ ) have beneficial effects on skin quality in mature women. In particular, $\mathrm{CH} 2$, which has a higher molecular weight $(4 \mathrm{kDa})$, demonstrated the greatest range if these effects including improvement of skin biomechanics, decrease of wrinkles, good subject satisfaction and no related adverse events. In addition, $\mathrm{CH} 3$ also presented certain interesting activities and $\mathrm{CH} 1$ expressed a significant effect on skin biomechanics. An extension of the treatment duration to three months or more could confirm these results and enhance the effects.

Acknowledgements: The study was funded by Weishardt International Group (France) which provided the fish collagen peptides Naticol® assessed in this study.

Conflict of interest: L. Duteil, C. Queille-Roussel, Y. Maubert and J. Esdaile belong to the investigation team which performed this study. They did not receive direct funding from Weishardt. C. Bruno-Bonnet is employed by Weishardt. J.P. Lacour has no conflict of interest with Weishardt.

Ethical Standards: The study protocol was approved by the Ethics Committee of Nice (France).

\section{References}

1. Kohl E, Steinbauer J, Landthaler M et al. Skin ageing. J Eur Acad Dermatol Venereol 2011;25(8), 873-84

2. Kyung AH, Bo-Rim Y, Kyung-Chul C.Molecular Mechanisms and In Vivo Mouse Models of Skin Aging Associated with Dermal Matrix Alterations. Lab Anim Res 2011;27(1), 1-8

3. Makrantonaki E, Zouboulis CC, William J. Characteristics and pathomechanisms of endogenously aged skin. Dermatology 2007;214, 352360

4. Gilchrest BA.Photoaging. J Invest Dermatol 2013;133(E1):E2-6

5. Uitto J.Understanding premature skin aging. N Engl J Med 1997;337, 14631465

6. Uitto J, Fazio M, Olsen DR.Molecular mechanisms of cutaneous aging. J Am Acad Dermatol 1989;21, 614-622

7. Oikarinen A, Karvonen J, Uitto J et al.Connective tissue alterations in skin exposed to natural and therapeutic UV-radiation. Photodermatol 1985;2, 115126

8. Lovell CR, Smolenski KA, Duance VC et al.Type I and III collagen content and fibre distribution in normal human skin during ageing. Br J Dermatol 1987;117, 419-428

9. Birk D E and Trelstad RL.Extracellular compartments in tendon morphogenesis. collagen fibril, bundle, and macroaggregate formation. J Cell Biol 1986;103, 231-240

10. Mendler M, Eich-Bender SG, Vaughan L et al. Cartilage contains mixed fibrils of collagen types II, IX and XI. J Cell Biol 1989;108, 191-198
11. Wasswa J, Tang J, Gu X. Utilization of fish processing by-products in the gelatin industry. Food Reviews International 2007;23, 159-174

12. Ferraro V, Cruz IB, Ferreira Jorge R et al. Valorisation of natural extracts from marine source focused on marine by-products: A review. Food Research International 2010;43, 2221-2233

13. Young S, Wong M, Tabata $Y$ et al. Gelatin as a delivery vehicle for the controlled release of bioactive molecule. Journal of Controlled Release 2005;109, 256-274

14. Wang L, An X, Yang F et al. Isolation and characterization of collagens from the skin, scale and bone of deep-sea redfish (Sebastes mentella). Food Chemistry 2008;108, 616-623

15. Baziwane D and He Q. Gelatin: the paramount food additive. Food Reviews International 2003;19(4), 423-435

16. Sumida E, Hirota A, Kuwaba K et al. The effect of oral ingestion of collagen peptide on skin hydration and biochemical data of blood. J Nutr Food 2004;7, $45-52$

17. Wu J, Fujioka M, Sugimoto K. Assessment of effectiveness of oral administration of collagen peptide on bone metabolism in growing and mature rats. J Bone Miner Metab 2004;22, 547-553

18. Matsuda N, Koyama Y, Hosaka Y et al. Effects of ingestion of collagen peptide on collagen fibrils and glycosaminoglycans in the dermis. J Nutr Sci Vitaminol (Tokyo) 2006;52, 211-215

19. Matsumoto $\mathrm{H}$, Ohara $\mathrm{H}$, Ito $\mathrm{K}$ et al. Clinical effects of fish type 1 collagen hydrolysate on skin properties. ITE Letters on batteries, new technologies and medicine 2006;7(4), 386-90

20. Zague $\mathrm{V}$ A. new view concerning the effects of collagen hydrolysate intake on skin properties. Arch Dermatol Res 2008;300, 479-483

21. Shigemura Y, Kubomura D, Sato Y et al.Dose-dependent changes in the levels of free and peptide forms of hydroxyproline in human plasma after collagen hydrolysate ingestion. Food Chemistry 2014159, 328-332

22. Proksch E, Segger D, Degwert J et al. Oral supplementation of specific collagen peptides has beneficial effects on human skin physiology: a doubleblind, placebo controlled study. Skin Pharmacol Physiol 2014;27, 47-55

23. Lemperle G, Holmes RE, Cohen SR et al.A classification of facial wrinkles. Plast Reconstr Surg 2001;1735-1752

24. Ryu HS, Joo YH, Kim SO et al.Influence of age and regional differences on skin elasticity as measured by the Cutometer ${ }^{\circledR}$. Skin Research and Technology 2008;14, 354-358

25. Krueger N, Luebberding S, Oltmer $M$ et al.Age-related changes in skin mechanical properties: a quantitative evaluation of 120 female subjects. Skin Research and Technology 2011;17, 141-148

26. Clarys P, Clijsen R, Barel A.Influence of probe application pressure on in vitro and in vivo capacitance (Corneometer CM 825®) and conductance (Skicon 200 EX®) measurements. Skin Research and Technology 2011;17(4), 445-450

27. Berardesca E. EEMCO guidance for the assessment of stratum corneum hydration: electrical methods. Skin Research and Technology 1997;3(2), 126132

28. Jiang LI, Stephens TJ, Goodman R.SWIRL, a clinically validated, objective, and quantitative method for facial wrinkle assessment. Skin Research and Technology 2013;0, 1-7

29. Yaar M, Eller M.S, Gilchrest BA. FiftyYears of Skin Aging. JID Symposium Proceedings 2002;7, 51-58

30. Mukherjee S, Date A, Patravale V et al. Retinoids in the treatment of skin aging: an overview of clinical efficacy and safety. Clinical Interventions in Aging 2006;1(4), 327-348

31. Kang S, Fisher G, Voorhees JJ. Photoaging: Pathogenesis, prevention and treatment. Clin Ger Med 2001;17, 643-59

32. Gilchrest BA. A review of skin ageing and its medical therapy. Br J Dermatol 1996;135(6), 867-75

33. Rittié L and Fisher G. UV-light-induced signal cascades and skin aging. Aging Res Rev 2002;1, 705-20

34. Minaguchi J, Koyama Y, Meguri N et al. Effects of ingestion of collagen peptide on collagen fibrils and glycosaminoglycans in Achilles tendon. J Nutr Sci Vitaminol 2005;51(3), 169-74

35. Hou H, Li B, Zhao X et al. The effect of pacific cod (Gadus macrocephalus) skin gelatin polypeptides on UV radiation-induced skin photoaging in ICR mice. Food Chemistry 2009;115 945-950

36. Ohara H, Ichikawa S, Matsumoto H et al. Collagen-derived ipeptide, prolinehydroxyproline, stimulates cell proliferation and hyaluronic acid synthesis in cultured human dermal fibroblasts. J Dermatol 2010;37(4), 330-338

37. Zague V, de Freitas V, da Costa Rosa M et al. Collagen hydrolysate intake increases skin collagen expression and suppresses matrix metalloproteinase 2 activity. J Med Food 2011;14, 618-624

38. Tanaka M, Koyama Y, Nomura Y. Effects of collagen peptide ingestion on UV-B-induced skin damage. Biosci Biotechnol Biochem 2009;73(4), 930-932

39. Zhuang Y, Sun L, Zhao X et al. Antioxidant and melanogenesisinhibitory activities of collagen peptide from jellysfish (Rhopilema esculentum). Journal of the Science of Food and Agriculture 2009;89, 1722-1727

40. Zhang Z, Zhao M, Wang J et al. Oral administration of marine collagen peptides from Chum Salmon skin enhances cutaneous wound healing and angiogenesis in rats. J Sci Food Agric 2011;91(12), 2173-2179 
41. Zhang Z, Wang J, Ding Y et al. Oral Administration of Skin Gelatin Isolated from Chum Salmon (Oncorhynchus keta) Enhances Wound Healing in Diabetic Rats. Mar Drugs 2011;9, 696-711

42. Liang J, Pei X, Zhang Z et al. The protective effects of long-term oral administration of marine collagen hydrolysate from chum salmon on collagen matrix homeostasis in the chronological aged skin of SpragueDawley male rats. J Food Sci 2010;75(8), 230-8

43. Zhuang $\mathrm{Y}, \mathrm{Hou} \mathrm{H}, \mathrm{Zhao} \mathrm{X}$ et al. Effects of collagen and collagen hydrolysate from jellyfish (Rhopilema esculentum) on mice skin photoaging induced by UV irradiation. J Food Sci 2009;74, H183-H188
44. Schwartz SR, Park J. Ingestion of BioCell Collagen(®), a novel hydrolyzed chicken sternal cartilage extract; enhanced blood microcirculation and reduced facial aging signs. Clinical Interventions in Aging 2012;7:267-273. 